\title{
Comparative Study of Modifying Meatal Advancement Glandular with Release Chordi versus Snodgrass Surgical Methods Regarding the Repair of Distal Hypospadias
}

\author{
Shahnam Askarpour ${ }^{1}$, Mehran Peyvasteh $^{1}$, Armin Mohamadi ${ }^{{ }^{*}}$, Mahmoud Khoshkhabar ${ }^{1}$
}

1. Department of Surgery, Ahvaz Jundishapur University of Medical Sciences, Ahvaz, Iran

*Corresponding Author:

Dr Armin Mohamadi

Department of Surgery, Ahvaz Jundishapur University of Medical Sciences, Ahvaz, Iran.

Email: armin_my_4804@yahoo.com

Received: 14 May 2021

Revised: 10 Sep 2021

accepted: 26 Sep 2021

\section{ABSTRACT}

\section{BACKGROUND}

Hypospadias is one of the most common congenital anomalies of the external genitalia of boys. No single technique can be recommended for the repair of hypospadias in its various forms. We aimed to compare modify meatal advancement glandular with release chordi versus Snodgrass surgical methods in the repair of distal hypospadias.

\section{METHODS}

In this study, conducted from Apr 2018 to the end of Sep 2020, all boys who underwent one of the two methods of Snodgrass and modify meatal advancement glanuplasty with release chordi in Imam Khomeini and Abuzar Hospitals of Ahvaz, Southern Iran, were enrolled.

\section{RESULTS}

Forty-five patients underwent Snodgrass (group S) and 55 patients underwent modified meatal advancement glandular with release chordi (group M). The mean age of patients and duration of surgical wound healing in the two groups did not differ significantly. There was no significant difference between the two groups in terms of complications, including Bleeding, Hematoma, Meatus stricture, Wound infection, detachment of the wound edge, chordi after surgery, Balanitis and Urethral stricture but the incidence of fistula in patients undergoing Snodgrass repair was significantly higher than the group modify meatal advancement glandular with release chordi $(\mathrm{P}<0.05)$.

\section{CONCLUSION}

The method of modify meatal advancement glandular with release chordi compared to Snodgrass method is associated with fewer complications due to surgery, although further studies are recommended.

\section{KEYWORDS}

Hypospadias; Snodgrass; Modify meatal advancement glanuplasty.

\section{Please cite this paper as:}

Askarpour Sh., Peyvasteh M., Mohamadi A., Khoshkhabar M. Comparative Study of Modifying Meatal Advancement Glandular with Release Chordi versus Snodgrass Surgical Methods Regarding the Repair of Distal Hypospadias. World J Plast Surg. 2021;10(2):73-77.

doi: 10.29252/wjps.10.3.73 


\section{INTRODUCTION}

Hypospadias is one of the most common congenital anomalies of the external genitalia of boys, occurring in approximately one in every 250 infants or approximately one in every 125 live boys $^{1,2}$. Numerous classification systems have been introduced for hypospadias, all of which divide hypospadias into anterior (distal), medial, and posterior (proximal) types according to the location of the urinary meatus. Anterior hypospadias (distal) accounts for $50 \%$ of cases and includes glandular, coronal and subcoronal. The median type is $30 \%$ of patients and includes the distal penile, body, and proximal penile, and the posterior type is $20 \%$ of cases including penoscrotal, scrotal and perineal ${ }^{3,4}$. Several surgical techniques for hypospadias repair have been developed that depend on the site of the urethral meatus in children ${ }^{1}$. However, no single method can be recommended to correct hypospadias, and surgeons traditionally divide hypospadias into distal and proximal defects. Based on reports, the outcomes of anterior hypospadias repair are desirable, with a low incidence of redo surgery, however complications were seen in up to $10 \%$ of subjects. Generally, postoperative complications often occur within 7-10 d following surgery in most cases. However, long-term followup is required due to delayed presentation of the urethral fistula and recurrent curvature of the penis after puberty ${ }^{5,6}$.

The TIP (Tubularized Incised Plate) or Snodgrass technique was introduced in 1994 in terms of the simple Thiersch-Duplay technique, which involved urethral tubing. A critical step in the Snodgrass technique is cutting through the midline of the urethral plate, which widens a narrow plate into a square in the depths of the urethra, resulting in a new slit nermeatus and a new normal diameter urethra ${ }^{7}$. Although this technique is easy to perform and has good aesthetic and functional results, several complications have been reported, the most common of which is meatal/neorethral stenosis, for which several causes have been suggested. During repair, technical problems include making a meatus with a very narrow duct or rigid glanuplasty, ischemia or xerotica narrowing balanitis, and the most important cause of scarring following posterior urethral incision are some of the causes of this complication ${ }^{5}$.
The technique of meatal advancement glanuplasty (MAGPI) was first described in 1981 by Duckett ${ }^{8}$. In 2002, the MAGPI method was modified, which became known as modify meatal advancement glandular technique with release chordi. Using the principles of maximal urethral lifting with a length of $2.5-2 \mathrm{~cm}$, by releasing the urethra, it reached the level of the bulbar and used the BEAM method for urethroplasty 9 .

In fact, the distal hypospadias repair techniques should be simple and result in functional benefits and satisfactory outcomes. Despite the development of multiple repair methods, there is no acceptable approach for complications ${ }^{10}$. In different studies, comparisons have been made between the hypospadias repair technique, but considering that no study has compared these techniques so far, we decided to compare the two techniques in distal hypospadias repair by designing and implementing this study.

\section{MATERIAL AND METHODS}

In this study, conducted from Apr 2018 to the end of Sep 2020, all boys who underwent one of the two methods of Snodgrass and modify meatal advancement glanuplasty with release chordi in Imam Khomeini and Abuzar hospitals of Ahvaz, southern Iran, were enrolled. The sampling method was census, and all patients who underwent distal hypospadias repair during our study were examined. Information including patient profile, age, time of operation, type of technique, postoperative bleeding, hematoma, meatus stricture, Fistula, wound infection, Detachment of the wound edge, chordi after surgery, balanitis and urethral stricture contained in their file were extracted. Obviously, those patients whose examination results in the file are incomplete or distorted were excluded from the study.

The study was approved by the Ethics Committee of Ahvaz Jundishapur University of Medical Sciences (IR.AJUMS.HGOLESTAN.REC.1399.061). The written informed consent was obtained from all parents of patients, their parents, or legal guardian.

\section{Statistical Analysis}

Data were analyzed using SPSS software (ver. 24, Chicago, IL, USA). To determine the normal or abnormal distribution of quantitative data, the 
Kolmogorov-Smirnov test was used. Independent $t$ test was used to compare the mean of data with normal distribution and Mann-Whitney test was used for data with abnormal distribution. On the other hand, Chi-square test and Fisher's exact test were used to compare qualitative data. $P$-value less than 0.05 was considered significant.

\section{RESULTS}

One hundred patients were enrolled. Forty-five patients underwent Snodgrass (group S) and 55 patients underwent modified meatal advancement glandular with release chordi (group M) (Figure 1 and 2). The mean age of patients in the two groups was compared with each other, there was no significant difference (Table 1).

The frequency of each postoperative complication in groups $(\mathrm{M})$ and $(\mathrm{S})$ was compared with each other (Table 2).

According to Table 2, the only complication that differed significantly between the two groups was
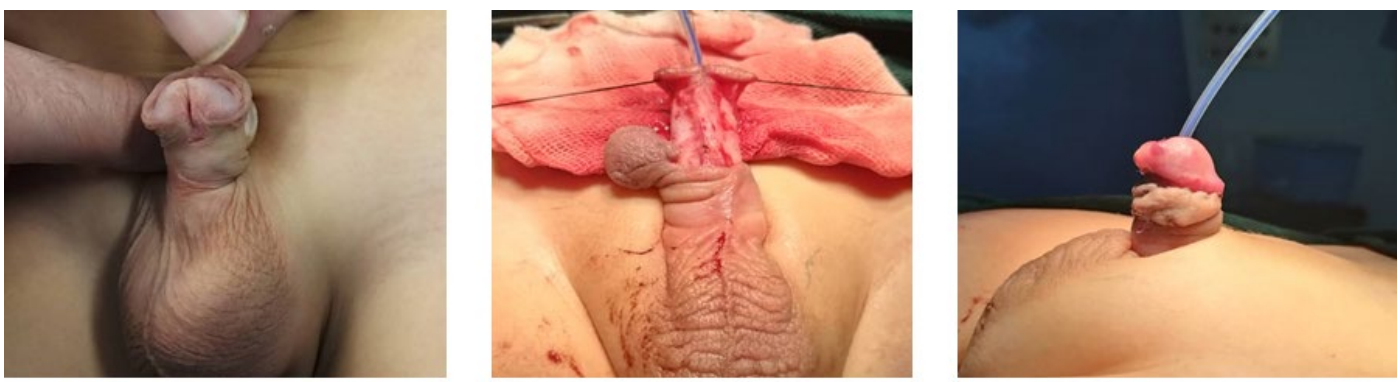

Fig. 1: Snodgrass technique for hypospadias repairing
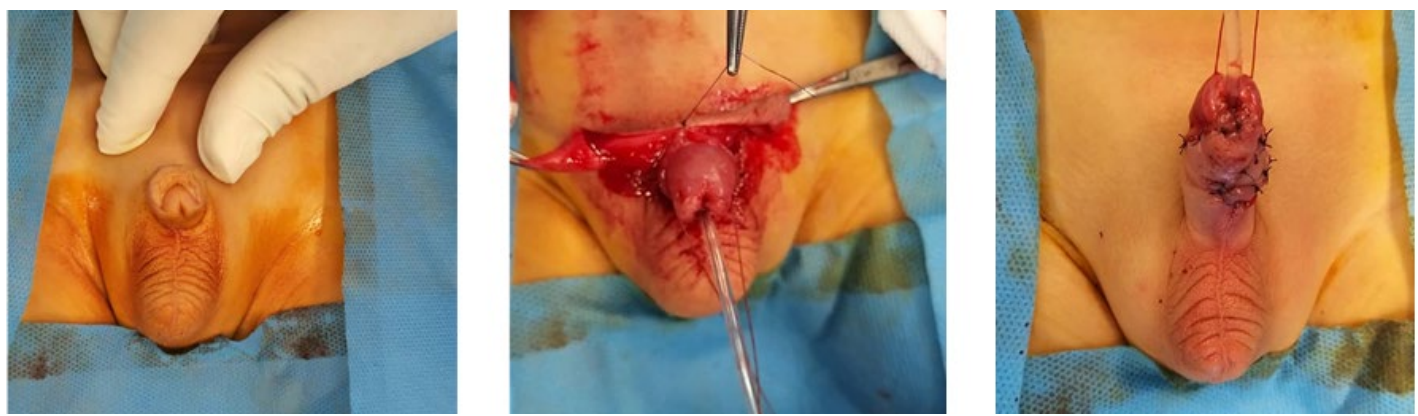

Fig. 2: Modify meatal advancement glandular with release chordi for hypospadias repairin

Table 1: Comparison of mean age of patients in the two groups

\begin{tabular}{cccc}
\hline \multicolumn{4}{c}{ Age (yr) Mean (SD) } \\
\hline Group (S) & Group (M) & Test & $P$-value \\
$6.08(2.54)$ & $5.86(2.22)$ & Independent- $t$ & 0.649 \\
\hline
\end{tabular}

Table 2: Comparison of the frequency of postoperative complications in groups (M) and (S) with each other

\begin{tabular}{lcccc}
\hline Complications & Group (S) & Group (M) & Test & $P$-value \\
\hline Bleeding & $1(2.2)$ & $2(3.6)$ & Fisher's exact & 1.000 \\
Hematoma & $2(4.4)$ & $3(5.5)$ & Fisher's exact & 1.000 \\
Meatus stricture & $2(4.4)$ & $1(1.8)$ & Fisher's exact & 0.587 \\
Fistula & $10(22.2)$ & $4(7.3)$ & Fisher's exact & 0.043 \\
Wound infection & $2(4.4)$ & $1(1.8)$ & Fisher's exact & 0.587 \\
Detachment of the wound edge & $2(4.4)$ & $1(1.8)$ & Fisher's exact & 0.587 \\
chordi after surgery & $3(6.7)$ & $1(1.8)$ & Fisher's exact & 0.324 \\
Balanitis & $1(2.2)$ & $2(3.6)$ & Fisher's exact & 1.000 \\
Urethral stricture & $3(6.7)$ & $0(0)$ & Fisher's exact & 0.088 \\
\hline
\end{tabular}


Table 3: Comparison of mean duration of surgical wound healing of patients

\begin{tabular}{cccc}
\hline \multicolumn{3}{c}{ Healing duration (d) } & \\
& Mean (SD) & & \\
\hline Group (S) & Group (M) & Test & $P$-value \\
$14.17(3.29)$ & $13.98(2.79)$ & Independent- $t$ & 0.748 \\
\hline
\end{tabular}

fistula, which had a significantly higher incidence of fistula in group $S$ than group $M$. The mean duration of surgical wound healing of patients in the two groups was compared with each other, there was no significant difference (Table 3).

\section{DISCUSSION}

Hypospadias remains a challenging problem for surgeons. Various surgical techniques have been described for repairing anterior hypospadias MAGPI, Snodgrass, Mathieu, Arap, Mustard, and Barcat, among which MAGPI and Snodgrass are the most commonly used methods worldwide ${ }^{11}$. The current concept in hypospadias surgery is based on complete one-step repair of the deformity and should lead to functional excellence and a normal penis 5 .

Although over 300 surgical techniques are available for hypospadias repair, treatment is still challenging because of related complications. Based on studies, potential long-term sequelae of hypospadias repair are reported in $30 \%$ of these surgical approaches globally ${ }^{12}$. In the present study, 100 patients in the two groups were compared. The mean age of patients in the two groups did not differ significantly. The mean duration of surgical wound healing was not significantly different between the two groups. On the other hand, there was no significant difference between the two groups in terms of complications, including bleeding, hematoma, meatus stricture, wound infection, detachment of the wound edge, chordi after surgery, balanitis and urethral stricture. On the other hand, the incidence of fistula in patients undergoing Snodgrass repair was significantly higher than the group modify meatal advancement glandular with release chordi. In general, one of the common complication of hypospadias repair is fistula. The incidence of fistula formation following hypospadias repair has estimated $4 \%-25 \%{ }^{13}$. In this regard, the most complication due to Snodgrass method, fistula was reported, which is in line with the results of the present study ${ }^{14}$. On the other hand, the incidence of fistula after repair by modifying meatal glandular repair method was reported to be very small, which confirms the findings of the present study ${ }^{15}$.

According to our study, the method of modify meatal advancement glandular with release chordi compared to Snodgrass method is associated with fewer complications due to surgery. The successful outcome of hypospadias operation depends mainly on surgeons' experience, flap circulation, type of suture, type of instruments, and neourethral protecting cover ${ }^{11,16}$.

We achieved acceptable results for the both methods. However, the most important limitation in the following study is the limitation on sample size and retrospective sampling, so further studies in this field are recommended.

\section{CONCLUSION}

Modifying the meatal advancement glandular method showed a satisfactory outcome for hypospodias repairing compared to the Snodgrass method. The incidence of complications including Bleeding, Hematoma, Meatus stricture, Wound infection, Detachment of the wound edge, chordi after surgery, Balanitis and Urethral stricture are same between the two methods. Nonetheless, the incidence of fistula in the Snodgrass method is significantly higher than modify meatal advancement glandular method with release chordi. As a result, further studies and larger sample sizes to determine the premier technique in this area are recommended.

\section{ACKNOWLEDGEMENTS}

The authors are thankful of Clinical Research Development units, Imam Khomeini and Abuzar Hospitals for their assistance.

\section{CONFLICT OF INTERESTS}

The authors declare that they have no competing interests. 


\section{REFERENCES}

1. van der Zanden LF, van Rooij IA, Feitz WF, Franke B, Knoers NV, Roeleveld N. Aetiology of hypospadias: a systematic review of genes and environment. Hum Reprod Update 2012 May-Jun;18(3):260-83.

2. Springer A, Van Den Heijkant M, Baumann S. Worldwide prevalence of hypospadias. J Pediatr Urol 2016;12(3):152. e1-. e7.

3. Baskin LS. Hypospadias and urethral development. J Urol 2000 Mar;163(3):951-6.

4. Rübben I, Stein R. [Hypospadias : Insights and challenges]. Urologe A 2017 Oct;56(10):1256-65.

5. Alngaar Y, Alshahat O, Zayid T. A comparative study between Snodgrass and urethral advancement methods in the repair of distal penile Hypospadias. Al-Azhar International Medical Journal 2020.

6. Bhat A, Mandal AK. Acute postoperative complications of hypospadias repair. Indian J Urol 2008;24(2):241.

7. Snodgrass W, Bush N. Primary hypospadias repair techniques: A review of the evidence. Urol Ann 2016 OctDec;8(4):403-8.

8. Duckett JW, Snyder III HM. Meatal advancement and glanuloplasty hypospadias repair after 1,000 cases: avoidance of meatal stenosis and regression. J Urol 1992;147(3):665-9.
9. Taneli C, Genç A, Günsar C, et al. Modification of meatal advancement and glanuloplasty for correction of distal hypospadias. Scand J Urol Nephrol 2004;38(2):122-4.

10. Keays MA, Dave S. Current hypospadias management: Diagnosis, surgical management, and long-term patientcentred outcomes. Canadian Urological Association Journal 2017;11(1-2Suppl1):S48.

11. Hasoon MA. Comparative Study in Anterior Distal Hypospadias Reconstruction Utilizing Different Techniques (Mathieu and Snodgrass): Outcome, Complications and Failure Rate. IOSR J Pharm 2013;3:53-9.

12. Springer A, Krois W, Horcher E. Trends in hypospadias surgery: results of a worldwide survey. Europ Urol 2011;60(6):1184-9.

13. Holland A, Abubacker M, Smith G, Cass D. Management of urethrocutaneous fistula following hypospadias repair. Pediatr Surg Int 2008;24(9):1047.

14. Zhou Y, Lu J, Takahashi G. Snodgrass procedure for primary hypospadias repair. Int J Urol 2002;9(4):215-8.

15. Shukla AK, Singh AP, Sharma P, Shukla J. MAGPI technique for distal penile hypospadias; modifications to improve outcome at a single center. Arch Int Surg 2016;6(4):201.

16. Moradi M, Moradi Aa, Ghaderpanah F. Comparison of Snodgrass and Mathieu surgical techniques in anterior distal shaft hypospadias repair. Urol J 2005; 2(1):28-31. 